\title{
TORSION OF A FINITE ELASTIC CYLINDRICAL ROD PARTIALLY BONDED TO AN ELASTIC HALF SPACE*
}

\author{
BY \\ L. M. KEER (Northwestern University) \\ AND \\ N. J. FREEMAN (University of Miami)
}

\begin{abstract}
A solution is given for the problem of axially symmetric torsion of a finite elastic cylindrical rod partially bonded to an elastic half space. The problem is formulated in a manner that involves coupling between dual Dini series and dual integral equations. Auxiliary functions are introduced and the problem is reduced to the solution of a Fredholm integral equation of the second kind. Approximate closed-form results are obtained when the radius of the bonded region is less than one-half the radius of the cylinder. Fractional order singularities in the stress are noted and calculated for the case when the crack vanishes.
\end{abstract}

1. Introduction. The purpose of the present paper is to consider the problem of axially symmetric torsion of a finite, elastic cylinder of unit radius that is partially bonded to an elastic half space in a circular region $c<1$. This work is related to a recent paper by Sneddon, Srivastav and Mathur [1], who consider the problem of a finite cylindrical rod partially bonded to a rigid surface. In [1] dual Dini series are reduced to the determination of an auxiliary function from a Fredholm integral equation of the second kind, and a study of this equation was made. It was found that when $c<\frac{1}{2}$ the kernel in the integral equation could be neglected with no significant loss in accuracy. Since a similar integral equation arises from the formulation of the present problem, conclusions deduced from [1] are also of importance here.

When $c<1$, the stress singularity is always square root, and when $c<\frac{1}{2}$, the approximation of [1] may be applied to give closed-form results for quantities of physical interest such as stress, torque-twist, and strain energy. It is also possible to deduce the critical torque for crack propagation in this case. The special case of $c=1$ (no crack) is also discussed. Here, the integral equation becomes identical to one studied by Freeman and Keer [2]. It was suggested in [2] that singularities in stress of fractional order were likely to occur but no attempt was made to deduce their value. Westmann [3] in a discussion of [2] has calculated possible values of these singularities by using the method of Williams [4]. By considering an asymptotic expansion of the kernel it is shown that stress singularities arising from an investigation of the present integral equation are identical to those calculated by Westmann.

2. Formulation of problem. We consider a cylindrical rod of length $\delta$ (where the radius is taken as the unit of length) that is imperfectly bonded to an elastic half space. Cylindrical coordinates $\rho, \theta$, and $z$ are used and the coordinate system is located at the top of the $\operatorname{rod}(z=0,0 \leq \rho \leq 1)$. The top of the rod is assumed to undergo displacement

*Received January 22, 1968. Supported by the U. S. Army Research Office-Durham and the National Science Foundation. 
through an angle $\gamma$ by means of a rigidly attached disk. The half-space occupies the region $\delta \leq z<\infty$, and the rod-half-space system is perfectly bonded in the region $0 \leq \rho \leq c$, $z=\delta$. Since this problem is one of axially symmetric torsion, there will be no dependence upon $\theta$. The only displacement is

$$
\mathfrak{u}=\left(0, u_{\theta}, 0\right),
$$

the nonvanishing stresses are given by

$$
S_{z \theta}=\partial u_{\theta} / \partial z, \quad S_{\rho \theta}=\rho \partial / \partial \rho\left(\rho^{-1} u_{\theta}\right)
$$

and the only nontrivially satisfied equation of equilibrium is

$$
\partial^{2} u_{\theta} / \partial \rho^{2}+\rho^{-1} \partial u_{\theta} / \partial \rho-\rho^{2} u_{\theta}+\partial^{2} u_{\theta} / \partial z^{2}=0
$$

where $\sigma_{z \theta}=\mu S_{z \theta}, \sigma_{\rho \theta}=\mu S_{\rho \theta}$, and $\mu$ is the shear modulus.

It is desired to determine the displacement functions from the boundary value problem defined by Eq. (3) and the following boundary conditions:

$$
\begin{array}{cl}
\partial / \partial \rho\left(\rho^{-1} u_{\theta}\right)=0 & (\rho=1,0 \leq z \leq \delta) \quad \text { (curved surface of rod stress free) } \\
u_{\theta}=\gamma \rho & (z=0,0 \leq \rho \leq 1) \\
u_{\theta}^{*} \rightarrow 0 \text { as } & \left(\rho^{2}+z^{2}\right)^{1 / 2} \rightarrow \infty \\
u_{\theta}^{*}=u_{\theta} & (z=\delta, 0 \leq \rho \leq c) \\
\mu \partial u_{\theta} / \partial z=\mu^{*} \partial u_{\theta}^{*} / \partial z & (z=\delta, 0 \leq \rho \leq c) \quad \text { (continuity of stress) } \\
\mu^{*} \partial u_{\theta}^{*} / \partial z=0 & (z=\delta, c<\rho<\infty) \\
\mu & (\text { exposed surface of half-space stress free) } \\
\mu \partial u_{\theta} / \partial z=0 & (z=\delta, c<\rho<1) \\
& \text { (stress free portion of cylinder half-space interface) }
\end{array}
$$

where starred quantities refer to the half-space and unstarred quantities refer to the rod (e.g. $\mu^{*}$ is the half-space shear modulus), and $u_{\theta}, u_{\theta}^{*}$ are governed by (3).

3. Reduction of problem to integral equation. It is desired to reduce the boundary value problem defined by Eqs. (3-10) to an integral equation. The methods available for such a reduction are now somewhat standard and the analysis is similar to that given in [1], [2]. The displacements $u_{\theta}, u_{\theta}^{*}$ are given the following representations:

$$
u_{\theta}=\gamma \rho+b_{0} \rho z+\sum_{n=1}^{\infty} b_{n} J_{1}\left(\lambda_{n} \rho\right) \sinh \left(\lambda_{n} z\right) \quad(0 \leq z \leq \delta)
$$

where $\lambda_{n} J_{1}^{\prime}\left(\lambda_{n}\right)-J_{1}\left(\lambda_{n}\right)=J_{2}\left(\lambda_{n}\right)=0, n=1,2, \cdots$, and

$$
u_{\theta}^{*}=\int_{0}^{\infty} A(\xi) \exp [-\xi(z-\delta)] J_{1}(\xi \rho) d \xi \quad(\delta \leq z<\infty)
$$

where $(11)$ will satisfy $(3,4,5)$ and (12) will satisfy $(3,6)$.

By choosing the substitutions

$$
a_{n}=\mu \lambda_{n} b_{n} \cosh \left(\lambda_{n} \delta\right) / \mu^{*}, \quad a_{0}=\mu b_{0} / \mu^{*}, \quad \alpha=-\mu^{*} / \mu, \quad \bar{\alpha}=\delta \alpha
$$


and using the approximation ${ }^{1}$

$$
\tanh \left(\lambda_{n} \delta\right) \cong 1
$$

the remaining equations, (7-10), may be written in the form:

$$
\begin{array}{rlrl}
\bar{\alpha} a_{0} \rho+\alpha \sum_{n=1}^{\infty} \lambda_{n}^{-1} a_{n} J_{1}\left(\lambda_{n} \rho\right)+\int_{0}^{\infty} A(\xi) J_{1}(\xi \rho) d \xi=\gamma \rho & & (0 \leq \rho \leq c) \\
a_{0} \rho+\sum_{i=1}^{\infty} a_{n} J_{1}\left(\lambda_{n} \rho\right)+\int_{0}^{\infty} \xi A(\xi) J_{1}(\xi \rho) d \xi & =0 & & (0 \leq \rho \leq c) \\
\int_{0}^{\infty} \xi A(\xi) J_{1}(\xi \rho) d \xi & =0 & & (c<\rho<\infty) \\
a_{0} \rho+\sum_{n=1}^{\infty} a_{n} J_{1}\left(\lambda_{n} \rho\right) & =0 & & (c<\rho<1) .
\end{array}
$$

Following techniques given in Copson [5] and Srivastav [6], the function $A(\xi)$ and the series (18) are given the representations below:

$$
\begin{gathered}
A(\xi)=(\pi / 2)^{1 / 2} \xi^{1 / 2} \int_{0}^{c} t^{1 / 2} g(t) J_{1 / 2}(\xi t) d t \\
a_{0} \rho+\sum_{n=1}^{\infty} a_{n} J_{1}\left(\lambda_{n} \rho\right)=\frac{d}{d \rho} \int_{\rho}^{c} g(t)\left(t^{2}-\rho^{2}\right)^{-1 / 2} d t H(c-\rho)
\end{gathered}
$$

where $H(x)$ is Heaviside's step function. The Dini coefficients in (20) may be solved for directly as

$$
\begin{aligned}
& a_{0}=-8 \int_{0}^{c} t g(t) d t \\
& a_{n}=-2\left[J_{1}\left(\lambda_{n}\right)\right]^{-2} \int_{0}^{c} \sin \left(\lambda_{n} t\right) g(t) d t .
\end{aligned}
$$

The results, when put back into $(15,16)$, lead to the following integral equation:

$$
g(t)=\chi(t)+\bar{\beta} \int_{0}^{c} L(t, u) g(u) d u
$$

where $\chi(t)=4 \gamma t / \pi, \bar{\beta}=\mu^{*} / \mu$ and

$$
L(t, u)=-\frac{4}{\pi}\left\{8 \delta t u+\sum_{n=1}^{\infty} \frac{\sin \left(\lambda_{n} t\right) \sin \left(\lambda_{n} u\right)}{\lambda_{n} J_{1}^{2}\left(\lambda_{n}\right)}\right\} .
$$

Equations $(23,24)$ are identical to Eqs. $(20,21)$ in [2] except for the limit on the integral. Equation (23) is a Fredholm integral equation of the second kind. Using the following integral representation

$$
\begin{aligned}
\sum_{n=1}^{\infty} \frac{\sin \left(\lambda_{n} t\right) \sin \left(\lambda_{n} u\right)}{\lambda_{n} J_{1}^{2}\left(\lambda_{n}\right)}=\frac{\pi}{4} \frac{t}{u} \delta(u & -t)-4 t u \\
& -\frac{1}{\pi} \int_{0}^{\infty} \frac{K_{2}(y)}{I_{2}(y)}\left[8 t u I_{2}(y)-\sinh (t y) \sinh (u y)\right] d y
\end{aligned}
$$

'The approximation (14) implies that the rod is "long." For all practical purposes this means that $\delta>1.4$. 
developed in [2], where $\delta(x)$ is Dirac's delta function, and introducing the function $G(t)$ as

$$
G(t)=(1+\bar{\beta}) g(t) /\left[\frac{4 \gamma}{\pi}+\frac{16 \bar{\beta}}{\pi}(1-2 \delta) \int_{0}^{c} u g(u) d u\right],
$$

(23) becomes

$$
G(t)=t+\omega \int_{0}^{c} M(t, u) G(u) d u
$$

where $\omega=\bar{\beta} /(1+\bar{\beta})$ and

$$
M(t, u)=\frac{4}{\pi^{2}} \int_{0}^{\infty} \frac{K_{2}(y)}{I_{2}(y)}\left[8 t u I_{2}(y)-\sinh (t y) \sinh (t u)\right] d y
$$

for $0 \leq \bar{\beta} \leq \infty$. The remainder of the paper will be concerned with the determination of significant physical quantities. It is appropriate to remark here that equation (27), except for the constant $\omega \leq 1$, is identical to (2.7) in [1] and as $\bar{\beta} \rightarrow \infty$ identity is obtained.

4. Stress, torque, and stress singularity. The results in this section and in the one to follow will be given in a closed form representation. This is possible due to the observation made in [1] that when $c<\frac{1}{2}$ the kernel in their equation (2.7) was negligible compared to other terms. Thus if $c$ is sufficiently small the approximation, when valid in [1], will certainly be valid here, since the present integral equation has its kernel multiplied by $\omega \leq 1$. In particular, the torque-twist relation in [1] was found to differ from an exact result by less than $1 \%$ when $c<\frac{1}{2}$, and the kernel was ignored. In the following the kernel will be neglected and certain physical results based upon this approximation will be obtained.

Using (2), (12), and (19) the stress at the interface can be shown to be

$$
S_{z \theta}=\bar{\beta} \frac{d}{d \rho} \int_{\rho}^{c} g(t)\left(t^{2}-\rho^{2}\right)^{-1 / 2} d t \quad(z=\delta) .
$$

If the kernel in (27) is ignored, then $g(t)$ may be written approximately as

$$
y(t)=\bar{\psi} t
$$

where

$$
\bar{\psi}=4 \gamma / \pi(1+\bar{\beta}-\psi), \quad \psi=16 \bar{\beta}(1-2 \delta) c^{3} / 3 \pi .
$$

Thus the stress may be written in the form

$$
S_{z \theta}=-\bar{\beta} \bar{\psi} \rho\left(c^{2}-\rho^{2}\right)^{-1 / 2} \quad(z=\delta) .
$$

From (29) the torque may be obtained as follows:

$$
T=4 \pi \mu^{*} \int_{0}^{c} t g(t) d t
$$

or using (30), as

$$
T=16 \gamma \mu^{*} c^{3} / 3(1+\bar{\beta}-\psi) .
$$

The St. Venant torque $T_{c}$ is given as

$$
T_{c}=\pi \mu \gamma / 2 \delta
$$


Defining the ratio $\tau$ as $\tau=T / T_{c}$, it is seen that

$$
\tau=32 \delta \bar{\beta} c^{3} / 3 \gamma(1+\bar{\beta}-\psi)
$$

and as $\bar{\beta} \rightarrow \infty$ equation (3.5) in [1] is obtained.

From equations $(12,30)$ the displacement may be calculated as

$$
u_{\theta}=\gamma \rho /(1+\bar{\beta}-\psi) \quad(z=\delta)
$$

and thus the displacement to the approximation given is linear.

Of significance is the stress intensity factor $N$, which is defined as

$$
S_{z \theta}\left(c^{-}\right)=N / s^{1 / 2}, \quad s=c-\rho .
$$

Thus

$$
N=-\bar{\beta} \Psi(c / 2)^{1 / 2}=-4 \bar{\beta} \gamma(c / 2)^{1 / 2} / \pi(1+\bar{\beta}-\psi) .
$$

It is seen from (39) that $N(c)$ will increase, reach a maximum, and then decrease. That $N$ vanishes at $c=1$ will now be shown in the discussion of stress singularities for the case of no crack.

Attention is now directed to the specific case when $c=1$. In this case (27) or series representation (23) are no longer Fredholm integral equations since the kernel will possess a strong singularity at $t=1$. If $c<1$, then $g(c)$ will be a constant and the stress singularity is always square root. When $c=1$ the singularity has to be investigated by other means. If $g(t)$ is assumed to be of the form

$$
g(t)=A t\left(1-t^{2}\right)^{\prime}
$$

where $A, \nu$ are constants then the stress is of the form

$$
S_{z \theta}=B \rho\left(1-\rho^{2}\right)^{-1 / 2+\nu}
$$

where $B$ is a constant. An investigation of $(23,27)$ by means of a test function of the form (40) is thus suggested.

When the test function (40) is inserted into (23), the resultant equation may be written as

$$
A t\left(1-t^{2}\right)^{\nu}=(4 \gamma / \pi-\bar{A}) t-2^{\nu} \bar{\beta} A \Gamma(\nu+1) t^{1 / 2}\left[S_{1,-1, v+3 / 2,1 / 2,-\nu-1}^{*}(1, t)\right]
$$

where $\bar{A}$ is a constant and $S_{\alpha, \beta, \gamma, \delta}^{*}(u, v)$ is defined in Srivastav [6]. Equation (42) is operated on by $d / d t\left(t^{-1}\right)$ and the resultant equation may be written as

$$
\begin{aligned}
2 \nu t\left(1-t^{2}\right)^{\nu-1}=-2^{\nu+1} \bar{\beta} \Gamma(\nu+1) t^{-1 / 2} & {\left[2^{-\nu} t^{3 / 2}\left(1-t^{2}\right)^{\nu-1} / \Gamma(\nu)\right.} \\
& \left.\quad-\frac{1}{\pi} \int_{0}^{\infty} \frac{K_{2}(y)}{I_{2}(y)} I_{\nu+3 / 2}(y) I_{3 / 2}(t y) y^{1-\nu} d y\right] .
\end{aligned}
$$

The singularity present in the integral portion of (43) may be isolated by adding and subtracting the asymptotic form of the integrand as $\xi \rightarrow \infty$ to obtain

$$
\begin{aligned}
2 \nu t\left(1-t^{2}\right)^{\nu-1}=-2^{\nu+1} \bar{\beta} \Gamma(\nu+1) t^{-1 / 2} & \left\{2^{-\nu} t^{3 / 2}\left(1-t^{2}\right)^{\nu-1} / \Gamma(\nu)\right. \\
& \left.-\left(t^{-1 / 2} / 2 \pi\right) \int_{0}^{\infty} \exp [-y(1-t)] y^{-\nu} d y+\bar{H}(t)\right\}
\end{aligned}
$$


where $\bar{H}(t)$ is nonsingular as $t \rightarrow 1$. Equation (44) becomes (as $t \rightarrow 1$ )

$2^{\nu} \nu t(1-t)^{\nu-1}=2^{\nu+1} \bar{\beta} \Gamma(\nu+1)\left[\Gamma(1-\nu)(1-t)^{\nu-1} / 2 \pi-t^{3 / 2}(1-t)^{\nu-1} / 2 \Gamma(\nu)\right]$

+ nonsingular terms

and the result

$$
\sin (\pi \nu)=\bar{\beta} /(1+\bar{\beta})
$$

is obtained by equating terms with a like singularity. It is seen from (46) that $0 \leq \nu \leq \frac{1}{2}$ with the equalities $\nu=0, \frac{1}{2}$ corresponding to the limiting cases of Reissner-Sagoci $(\bar{\beta}=0)$ and St. Venant torsion $(\bar{\beta}=\infty)$ respectively. Thus when $c=1, N=0$ and the stress singularities are of fractional order and have the values predicted by Westmann [3] using other techniques. A slightly modified method can be used to derive (46) from (27).

5. Strain energy and crack propagation. Information can be obtained concerning the influence of the crack when compared with an undamaged system if the approximation when $c<\frac{1}{2}$ used earlier is applied here. Using the value for torque given in (34), the strain energy $V=\frac{1}{2} T \gamma$ can be written as

$$
V=\frac{3 T^{2}}{32 \mu^{*} c^{3}}\left[1+\bar{\beta}\left(1+\frac{16 c^{3} \eta}{3 \pi}\right)\right]
$$

where $\eta=2 \delta-1$.

For the undamaged material an accurate approximation to the strain energy was given in [2] as

$$
V=\frac{T^{2}}{8 \mu^{*}(\pi)^{1 / 2} \Gamma}\left[1+\bar{\beta}\left(1+4 \Gamma \eta / \pi^{1 / 2}\right)\right]
$$

where $\Gamma=\Gamma(\nu+1) / \Gamma(\nu+5 / 2)$. Here $\nu$ depends upon $\bar{\beta}$ and values for $\bar{\beta}=1 / 3,1$ are given in [2]. Using $(47,48)$ and after some manipulation, $V$ is determined as

$$
V=\frac{T^{2}}{8 \mu^{*}(\pi)^{1 / 2} \Gamma}\left[1+\bar{\beta}\left(1+4 \eta \Gamma / \pi^{1 / 2}\right)\right]\left\{1+\frac{(1+\bar{\beta})\left[3 \pi^{1 / 2} \Gamma / 4 c^{3}-1\right]}{1+\bar{\beta}\left(1+4 \eta \Gamma / \pi^{1 / 2}\right)}\right\}
$$

where the second term on the right-hand side of (49) represents the increase $W$ in the strain energy. Thus

$$
W=\frac{(1+\bar{\beta}) T^{2}}{\mu^{*} \pi}\left\{\frac{3 \pi \Gamma-4(\pi)^{1 / 2} c^{3}}{32 c^{3} \Gamma}\right\}
$$

The surface energy of the crack is

$$
U=2 \pi\left(1-c^{2}\right) T_{0}
$$

and applying the criterion

$$
\partial(U-W) / \partial c=0,
$$

the critical torque is found to be

$$
T_{c}=(8 / 3)\left[2 \pi \mu^{*} c^{5} T_{0} /(1+\bar{\beta})\right]^{1 / 2} \quad(c<.5) .
$$

It is interesting to note that this value of critical torque is identical in form to that obtained by the authors [7] for the torsion of two finite, partially bonded cylinders. 


\section{REFERENCES}

[1] I. N. Sneddon, R. P. Srivastav, and S. C. Mathur, The Reissner-Sagoci problem for a long cylinder of finite radius, Quart. J. Mech. Appl. Math. 19, 123 (1966)

[2] N. J. Freeman and L. M. Keer, Torsion of a cylindrical rod welded to an elastic half space, J. Appl. Mech. 34, 687 (1967)

[3] R. A. Westmann, Discussion of [2], J. Appl. Mech. 35, 197 (1968)

[4] M. L. Williams, Stress singularities resulting from various boundary conditions in angular corners of plates in extension, J. Appl. Mech. 19, 526 (1952)

[5] E. T. Copson, On certain dual-integral equations, Proc. Glasgow Math. Assoc. 5, 21 (1961)

[6] R. P. Srivastav, Dual-series relations-II. Dual relations involving Dini series, Proc. Roy. Soc. Edin., A, 66, 173 (1964)

[7] N. J. Freeman and L. M. Keer, Torsion of elastic cylinders in contact, Int. J. Solids Structures 3, 799 (1967) 